\title{
Autosomal Recessive Hypophosphatemia Rickets
}

National Cancer Institute

\section{Source}

National Cancer Institute. Autosomal Recessive Hypophosphatemia Rickets. NCI

Thesaurus. Code C123187.

An autosomal recessive renal phosphate wasting disorder that results in rickets. 